\title{
Disruption of anchoring junctions in the testes of experimental varicocele rats
}

\author{
LIHONG ZHANG ${ }^{1-3}$, XIAOZHEN ZHAO ${ }^{1-3}$ and WEI WANG ${ }^{1-3}$ \\ ${ }^{1}$ Department of Human Anatomy, Histology and Embryology, School of Basic Medical Sciences, \\ Fujian Medical University; ${ }^{2}$ Key Laboratory of Aging and Neurodegenerative Disease, \\ School of Basic Medical Sciences; ${ }^{3}$ Laboratory of Clinical Applied Anatomy, \\ Fujian Medical University, Fuzhou, Fujian 350122, P.R. China
}

Received March 5, 2020; Accepted April 7, 2021

DOI: $10.3892 /$ etm.2021.10319

\begin{abstract}
Varicocele is a common disease of the male reproductive system and is the main cause of male infertility; however, the pathological mechanisms of varicocele remain unclear. The anchoring junctions (AJs) in the testies are located between Sertoli cells, or between Sertoli cells and germ cells. Intact and functional AJs are crucial for spermatogenesis. In the present study, the histomorphology, ultrastructure of AJ, cell cycle, expression of AJ structural proteins, and the level of AJ-associated signaling molecules were investigated in the left testes of experimental varicocele rats at 8 and 12 weeks after surgery. The results revealed that varicocele induced the loss of premature germ cells from the seminiferous epithelium. Furthermore, the results of the present study also revealed damage to the $\mathrm{AJ}$ ultrastructure, disorientation of the spermatid head, deregulation of the cell cycle, downregulation
\end{abstract}

Correspondence to: Professor Wei Wang or Professor Xiaozhen Zhao, Department of Human Anatomy, Histology and Embryology, School of Basic Medical Sciences, Fujian Medical University, 1 Xuefu North Road, University Town, Fuzhou, Fujian 350122, P.R. China

E-mail: 1401411234@qq.com

E-mail: zxz8338@163.com

Abbreviations: AJ, anchoring junction; ES, ectoplasmic specialization; BTB, blood-testis barrier; TJ, tight junction; FAK, focal adhesion kinase; MAPK, mitogen-activated protein kinase; Arp, actin-related protein; PI3K, phosphatidylinositol 3-kinase; SH2, Src homology domain 2; PI, propidium iodide; RIPA, radioimmunoprecipitation lysis buffer; PMSF, phenylmethanesulfonyl fluoride; PVDF, polyvinylidene difluoride membrane; H\&E, hematoxylin and eosin; PBS, phosphate-buffered saline; ECL, chemiluminescence; ERK, extracellular signal-regulated protein kinase; N-WASP, neuronal Wiskott-Aldrich syndrome protein; Cas, crk-associated substrate; PAK, p21-activated kinase; ER, endoplasmic reticulum

Key words: varicocele, anchoring junction, phosphorylated focal adhesion kinases of AJ structural proteins, enhanced phosphorylation of focal adhesion kinase (FAK) at $\mathrm{Tyr}^{397}$ and its downstream adapter Src at $\mathrm{Tyr}^{416}$, and activation of the extracellular signal-regulated protein kinase 1 (ERK1) signaling pathway. Thus, the present study demonstrated that varicocele disrupted the structure and function of AJs in the left testes of rats, and that enhancement of FAK phosphorylation may contribute to AJ damage by activating ERK1 signaling, disrupting actin-based filament networks, and altering the balance of the apical ectoplasmic specialization-blood testis barrier functional axis. These findings provide important insights into the pathological mechanisms through which varicocele contributes to male infertility and could help to identify new therapeutic targets for varicocele.

\section{Introduction}

There are two types of testis-specific cell-cell actin-based anchoring junctions (AJs) observed in the seminiferous epithelium, i.e., apical ectoplasmic specialization (ES) and basal ES (1). The apical ES exists between the interface of Sertoli cells and the step 8-19 spermatids in the adluminal compartment of the epithelium, using the cadherin/catenin, nectin/afadin, and integrin/laminin adhesion protein complexes as structural and functional units (2). Furthermore, the apical ES functions as the only anchoring installation to maintain spermatid polarity, provide the necessary nutrients and hormonal support for spermatids, and take part in signal transduction during spermiogenesis (3-5). The basal ES is located between Sertoli cells, and between Sertoli cells and spermatocytes in the basal compartment of the epithelium $(6,7)$; the former basal ES forms the blood-testis barrier (BTB) combined with tight junctions (TJs) and gap junctions, which build a specialized microenvironmental and immune barrier for spermatogenesis and permit the timely transit of preleptotene and leptotene spermatocytes at stage VIII. The dynamics of AJs can be regulated by several AJ-associated signaling molecules, including focal adhesion kinase (FAK), Src, Csk, cytokeratin 2, P120 ${ }^{\text {ctn }}$, small GTPases (e.g., RhoB, Rac1 and Cdc42) and cytokines, by affecting the phosphorylation status of AJ-associated structural protein complexes, the organization of the actin-based filament 
network, and the activation of signaling pathways related to actin polymerization and cell adhesion (6,8-12). Additionally, activation of the mitogen-activated protein kinase (MAPK) and phosphatidylinositol 3-kinase (PI3K)/Akt signaling pathways is involved in the disruption of AJs, likely via alterations to the organization of the actin filament-based cytoskeleton in the testes $(13,14)$. As a tight functional and structural link between TJs and AJs, the effectors of TJ dynamics are also important putative modulators of AJ dynamics (e.g., hormones and cytokines), acting in a direct or indirect manner.

FAK is a nonreceptor protein tyrosine kinase that is linked to occludin/zonula occludens-1 and integrin/laminin protein complexes, forming a functional unit at $\mathrm{TJ}$ and $\mathrm{AJ}$ sites, respectively, in the seminiferous epithelium $(14,15)$. In addition, FAK is involved in the regulation of $\mathrm{AJ}$ and $\mathrm{TJ}$ dynamics by switching its specific phosphorylated form (e.g., p-FAK-Tyr ${ }^{397}$, p-FAK-Tyr ${ }^{407}$ and p-FAK-Tyr ${ }^{576}$ ), which shows different localizations and functions in the seminiferous epithelium in vivo and in vitro $(5,16-19)$. For example, at the TJ site in vitro, p-FAK-Tyr ${ }^{397}$ and $\mathrm{p}-\mathrm{FAK}-\mathrm{Ty}^{407}$ work as an integrated component protein to regulate TJs, with the former promoting assembly and the latter facilitating disassembly of TJs by regulating the activation of actin-related protein (Arp) $2 / 3$ (19). At the apical ES in vivo, p-FAK-Tyr ${ }^{407}$ and p-FAK-Tyr ${ }^{397}$ are predominantly localized at the concave and convex sides of the spermatid heads in stage VII to early stage VIII, respectively. The former interacts with Arp 2/3, and the latter associates with $\alpha 6 \beta 1$-integrin, forming a functional unit during spermiation $(8,19)$. In addition, $\mathrm{p}-\mathrm{FAK}-\mathrm{Tyr}^{397}$ is the only phosphorylated form of FAK that provides a combined binding site for various signaling molecules, such as the Src homology domain 2 (SH2) (20) and PI3K (21), demonstrating involvement in integrin-initiated signaling pathways at the plasma membrane and various biological events.

Varicocele is the primary cause of male infertility and can be described as excessive dilatation of the spermatic vein. However, the mechanisms through which varicocele contributes to male infertility are remain unclear, and to the best of our knowledge, effective treatments have not yet been reported (22-26). In 1981, an experimental varicocele model was established through partial ligation of the left kidney vein (26). In addition, damage to the BTB, dysfunction of the neuroendocrine system, increased temperature, hypoxia, accumulation of metabolites and toxicants, and oxidative pressure are also involved in the pathological mechanisms of varicocele $(27,28)$. However, to the best of our knowledge, the disruption of AJs and the corresponding mechanisms induced by varicocele have not yet been reported.

Therefore, the present study evaluated the characteristics and changes to AJs in an experimental model of varicocele in rats. We aimed to identify new targets for the prevention and treatment of varicocele and for the improvement of male fertility.

\section{Materials and methods}

Reagents. The main reagents used in the present study were as follows: Propidium iodide (PI)/RNase Staining Buffer (BD Biosciences), horseradish peroxidase (HRP)-conjugated goat anti-rabbit IgG (cat. no. A0208; Beyotime Institute of Biotechnology), HRP-conjugated goat anti-mouse IgG (cat. no. IH-0031; Dingguo Changsheng Biotechnology Co., Ltd.), radioimmunoprecipitation lysis buffer (RIPA; Beyotime Institute of Biotechnology), phenylmethanesulfonyl fluoride (PMSF; Beyotime Institute of Biotechnology), prestained protein ladder (Thermo Fisher Scientific, Inc.), polyvinylidene difluoride (PVDF) membranes (EMD Millipore), enhanced chemiluminescence reagent (ECL; Beyotime Institute of Biotechnology), and Enhanced BCA Protein Assay Kit (Beyotime Institute of Biotechnology), bovine serum albumin (cat. no. ST023; Beyotime Institute of Biotechnology). The primary antibodies and working dilutions are presented in Table I.

Animals and surgical procedure. A total of 60 male

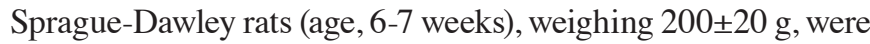
equally and randomly divided into four groups: i) The sham group for 8 weeks (Sham for 8 weeks); ii) the sham group for 12 weeks (Sham for 12 weeks); iii) the experimental varicocele group for 8 weeks (VC for 8 weeks); iv) and the experimental varicocele group for 12 weeks (VC for 12 weeks). Prior to surgery, all rats were housed in normal atmosphere $\left(\mathrm{N}_{2}, 78 \% ; \mathrm{O}_{2}, 21 \% ; \mathrm{CO}_{2}\right.$, $0.03 \%$ ) and specific pathogen-free controlled environmental conditions, with a 12-h day/night cycle, a temperature of $\sim 23^{\circ} \mathrm{C}$, a humidity of $40-70 \%$ and free access to standard rat food and water. As presented in Fig. 1, after $12 \mathrm{~h}$ fasting, the left renal vein of animals in the experimental varicocele model groups underwent partial ligation under anesthesia using $30 \mathrm{mg} / \mathrm{kg}$ sodium pentobarbital through intraperitoneal injection to establish the experimental left varicocele, as previously described (24), and the surgery lasted for $\sim 10 \mathrm{~min}$. Rats in the sham group underwent the same operation without the left vein ligation. Rats were housed alone following surgery, and were fully recovered after $\sim 7$ days. The penicillin was used to prevent infection via injection into the abdomen post-surgery. At 8 and 12 weeks after the operation, rats were sacrificed via cervical dislocation after being anesthetized using $30 \mathrm{mg} / \mathrm{kg}$ sodium pentobarbital via intraperitoneal injection to gain fresh left testicular tissue. Animals in the experimental varicocele model group who exhibited atrophic left kidney, or those that did not show vascular dilation, were excluded from subsequent experiments. A total of 30 rats that underwent surgery exhibited left vascular dilation without left kidney atrophying and were used to perform the subsequent experiment. The success rate of experimental varicocele model establishment in the present study was $100 \%$, and no animals died during the procedures. All animals were bred and maintained at the Laboratory Animal Center of Fujian Medical University (Fuzhou, Fujian, China). Rats were originally obtained from the National Seed Center of Experimental Rodent Animals (Beijing, China). The experimental procedures were performed in accordance with the Guidelines for the Care and Use of Laboratory Animals approved by Fujian Medical University (Animal Approval Committee no. \#SYXK-2012-0001).

Hematoxylin and eosin $(H \& E)$ staining. At 8 and 12 weeks after the operation, six animals from each group were anesthetized using $30 \mathrm{mg} / \mathrm{kg}$ sodium pentobarbital via intraperitoneal injection followed by cervical dislocation to obtain the left testes after cardiac perfusion using $500 \mathrm{ml}$ saline $(0.9 \%$ sodium chloride) and $500 \mathrm{ml} 10 \%$ formalin sequentially. The left testes 
Table I. Primary antibodies used in the western blot analysis.

\begin{tabular}{|c|c|c|c|}
\hline Antibody & Supplier & Cat. no. & Dilution \\
\hline Rabbit anti-N-cadherin & Elabscience Biotechnology, Inc. & ENT2988 & $1: 500$ \\
\hline Rabbit anti-E-cadherin & Elabscience Biotechnology, Inc. & ENT1454 & $1: 200$ \\
\hline Rabbit anti- $\alpha$-catenin & Elabscience Biotechnology, Inc. & ENT0669 & $1: 500$ \\
\hline Rabbit anti- $\beta$-catenin & Beijing Biosynthesis Biotechnology Co., Ltd. & bs- $1165 \mathrm{R}$ & $1: 200$ \\
\hline Rabbit anti- $\gamma$-catenin & Beijing Biosynthesis Biotechnology, Co., Ltd. & bs-6990R & $1: 200$ \\
\hline Rabbit anti-FAK & Cell Signaling Technology, Inc. & 3285 & $1: 500$ \\
\hline Rabbit anti-Phospho-FAK-Tyr ${ }^{397}$ & Cell Signaling Technology, Inc. & 8556 & $1: 500$ \\
\hline Rabbit anti-Src & Cell Signaling Technology, Inc. & 2108 & $1: 1,000$ \\
\hline Rabbit anti-phospho-Src-Tyr ${ }^{416}$ & Cell Signaling Technology, Inc. & 6943 & $1: 1,000$ \\
\hline Rabbit anti-ERK1/2 & Cell Signaling Technology, Inc. & 9102 & $1: 1,000$ \\
\hline Rabbit anti-phospho-ERK1/2 & Cell Signaling Technology, Inc. & 9101 & $1: 1,000$ \\
\hline$\beta$-actin mouse monoclonal antibody & Beyotime Institute of Biotechnology & AF0003 & $1: 1,000$ \\
\hline
\end{tabular}

FAK , focal adhesion kinase; ERK1, extracellular signal-regulated protein kinase 1.
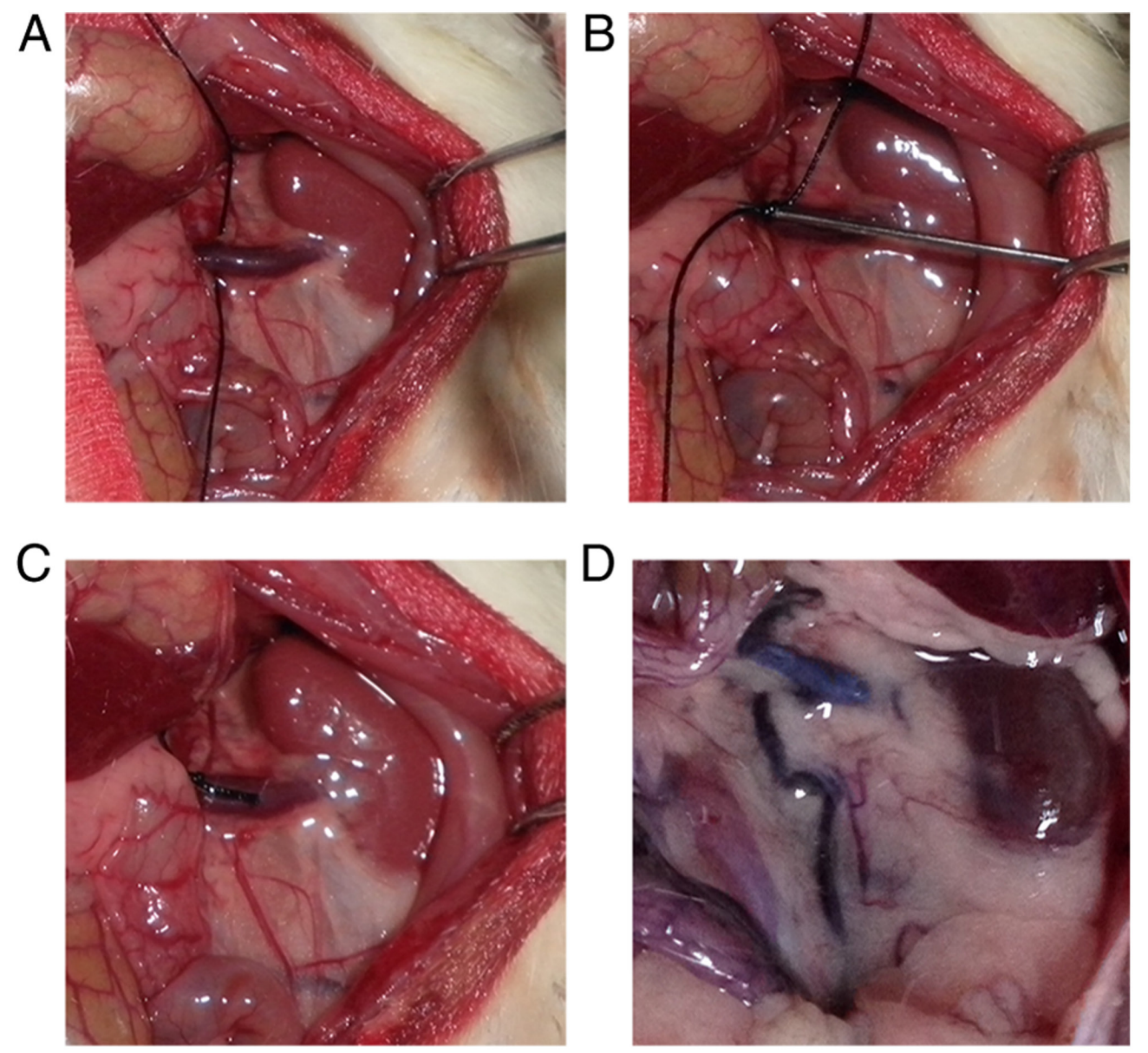

Figure 1. Establishment of experimental varicocele. (A-C) The procedure for left renal vein ligation with a $0.8 \mathrm{~mm}$ diameter needle and a 3-0 silk suture. (D) Varicose spermatic vein induced by experimental varicocele after 12 weeks.

were then removed and placed in $10 \%$ formalin solution for $24 \mathrm{~h}$ for post fixation. Next, the fixed left testicular tissues were embedded in paraffin, cut into 5- $\mu \mathrm{M}$-thick sections, set on poly-L-lysine-coated slides, stained with H\&E, and observed using a light microscope at different magnifications (x100, $\mathrm{x} 400$ and $\mathrm{x} 1,000)$ (29). The percentage of tubules containing deciduous immature germ cells was calculated by counting the positive tubules out of 50 seminiferous tubules from five to ten randomly selected fields for each sample.

Flow cytometry analysis. Dissociation of left testicular tissues was performed as previously described to obtain a single cell 

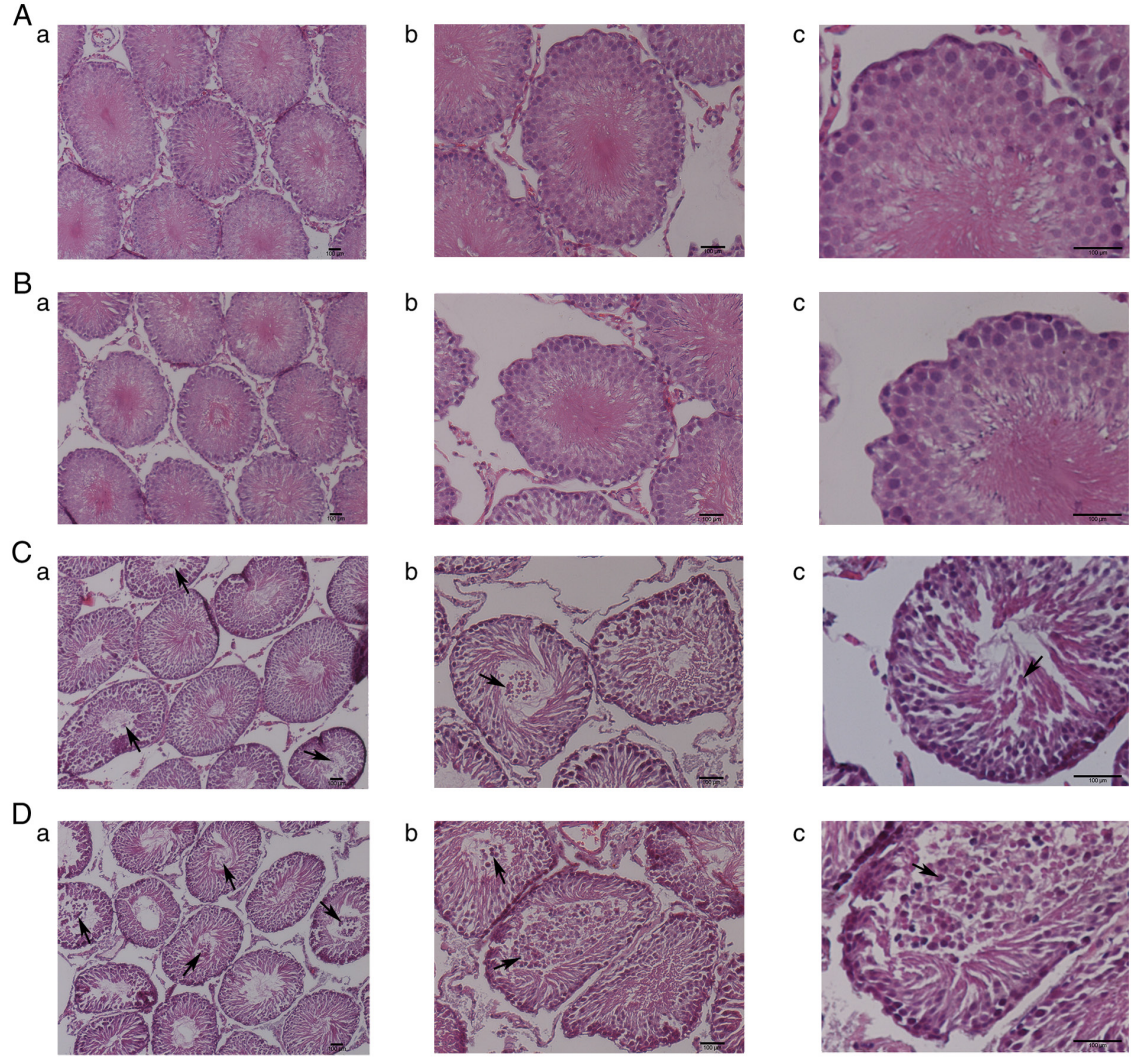

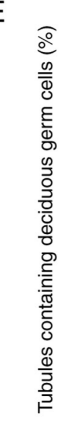



Figure 2. H\&E staining of the left testes. Representative H\&E images of the left testes of rats at different magnifications. Sham group for the (A) 8 and (B) 12 weeks, and the experimental varicocele group for the (C) 8 and (D) 12 weeks. The magnification of images is as follows: (a) x100, (b) x400 and (c) $\mathrm{x} 1,000$. These results are representative of six rats from each group. The black arrowheads in in C and D indicate the immature germ cells shedding from the seminiferous epithelium to the cavity. (E) The percentages of seminiferous tubules containing deciduous immature germ cells in the sham group and experimental VC group. The data are presented as the mean $\pm \mathrm{SD}(\mathrm{n}=6)$. ${ }^{*} \mathrm{P}<0.05$ vs. the sham group for 8 weeks; ${ }^{*} \mathrm{P}<0.05$ vs. the sham group for 12 weeks; ${ }^{* *} \mathrm{P}<0.05$. H\&E, hematoxylin and eosin; $\mathrm{VC}$, varicocele.

suspension for the flow cytometry analysis (30). Briefly, $\sim 10 \mathrm{mg}$ left testicular tissue from the six rats in each group was cut and removed in precooled phosphate-buffered saline (PBS) to remove the tunica albuginea and visible vessels. The tissue was then placed in $1 \mathrm{ml}$ digestion medium $(1 \mathrm{mg} / \mathrm{ml}$ collagenase, $1 \mathrm{mg} / \mathrm{ml}$ hyaluronidase and $1 \mathrm{mg} / \mathrm{ml}$ DNAse I in Dulbecco's modified Eagle's medium/F12) and minced with McPherson-Vannas scissors carefully until there were no obvious visible tissue masses. Next, the tissue digestion suspension was incubated at $37^{\circ} \mathrm{C}$ for $30 \mathrm{~min}$ with gentle rotation, followed by filtration with a 300-mesh nylon net to obtain a single-cell suspension. The suspension was centrifuged to collect single cells at 1,500 $\mathrm{x}$ g for 4 min at $4^{\circ} \mathrm{C}$. The cells were then washed with PBS three times, followed by the addition of $1 \mathrm{ml} \mathrm{75 \%}$ alcohol and incubation for $12 \mathrm{~h}$ at $4^{\circ} \mathrm{C}$ to fix the cells. Next, the $75 \%$ alcohol was removed via centrifugation at $1,500 \mathrm{x}$ g for $5 \mathrm{~min}$ at $4^{\circ} \mathrm{C}$, followed by washing the cells with PBS three times to remove any remaining alcohol. Lastly, $500 \mu \mathrm{l} \mathrm{PI} / \mathrm{RNase}$ Staining Buffer was added to each sample, and the reaction mixture was incubated at $37^{\circ} \mathrm{C}$ for $15 \mathrm{~min}$ in the dark. The cell cycle was analyzed using a flow cytometer (NovoCyte; Agilent Technologies, Inc.) with a 488-nm excitation laser and 575/26-nm bandpass filters for collection. NovoExpress 1.0.2 software (Agilent Technologies, Inc.) was used to analysis the data.

Transmission electron microscopy. Testicular tissue was obtained and cut into small pieces $(2 \times 2 \mathrm{~mm})$. The tissues were then immersed in $3 \%$ glutaraldehyde $/ 1.5 \%$ paraformaldehyde/0.1 M PBS ( $\mathrm{pH} 7.2)$ for at least $2 \mathrm{~h}$ at $4^{\circ} \mathrm{C}$. After washing with $0.1 \mathrm{M}$ PBS (pH 7.2) in triplicate, tissues were fixed in $1 \%$ osmium $/ 1.5 \%$ potassium ferrocyanide for $1.5 \mathrm{~h}$ at $4^{\circ} \mathrm{C}$, followed by washing with $0.1 \mathrm{M}$ PBS (p 7.2) in 
triplicate. The tissues were then dehydrated using 50\% alcohol for $10 \mathrm{~min}, 70 \%$ alcohol saturated uranium acetate solution for $12 \mathrm{~h}, 90 \%$ alcohol for $10 \mathrm{~min}, 90 \%$ alcohol $/ 10 \%$ acetone for $10 \mathrm{~min}, 90 \%$ acetone $10 \%$ alcohol for $10 \mathrm{~min}$ and $100 \%$ acetone for $10 \mathrm{~min}$. All dehydration procedures were performed at $4{ }^{\circ} \mathrm{C}$. The tissues were placed in $50 \%$ acetone $/ 50 \%$ epoxy resin $(618 / \mathrm{E}-51)$ for $1.5 \mathrm{~h}$ at $35^{\circ} \mathrm{C}$ and $100 \%$ epoxy resin (618/E-51) for $3 \mathrm{~h}$ at $35^{\circ} \mathrm{C}$. Subsequently, the tissues were embedded in epoxy resin $(618 / \mathrm{E}-51)$ for $12 \mathrm{~h}$ at $35^{\circ} \mathrm{C}, 12 \mathrm{~h}$ at $45^{\circ} \mathrm{C}$ and 3 days for $60^{\circ} \mathrm{C}$. The sections $(70-80 \mathrm{~nm})$ were stained using $2 \%$ uranium acetate for $5 \mathrm{~min}$ and lead citrate for $5 \mathrm{~min}$, both at room temperature, followed by washing with double distilled water in triplicate. The ultrastructure of the testis was observed using a transmission electron microscope (EM208 model; Philips Medical Systems, Inc.) with assistance from the laboratory staff of Fujian Medical University (Fuzhou, Fujian, China) trained in electron microscopy.

Western blot analysis. A total of $1 \mathrm{ml}$ RIPA lysis buffer with $10 \mu \mathrm{l}$ PMSF and $10 \mu \mathrm{l}$ phosphatase inhibitor cocktail was added to $100 \mathrm{mg}$ left testicular tissues to extract total protein on ice. The concentration of total protein was measured using Enhanced BCA Protein Assays. Proteins (40 $\mu \mathrm{g}$ per lane) were then separated via $8 \%$ SDS-PAGE. A molecular weight loading marker $(2 \mu 1)$ was used to determine the relative molecular weights of the proteins. The proteins on the gel were transferred to PVDF membranes, and the membranes were then blocked in $5 \%$ bovine serum albumin for $2 \mathrm{~h}$ at $4^{\circ} \mathrm{C}$, followed by incubation with the specific primary antibodies listed in Table I at $37^{\circ} \mathrm{C}$ for at least $12 \mathrm{~h}$. The membranes were then incubated with the corresponding horseradish peroxidase-conjugated goat anti-rabbit $\mathrm{IgG}$ and goat anti-mouse IgG secondary antibodies $(1: 1,000)$ for $2 \mathrm{~h}$ at $4^{\circ} \mathrm{C}$. Finally, the specific protein bands were detected using ECL reagent, and images were captured using a biological imaging system. ImageJ software (ImageJ 1.46r; National Institutes of Health) was used to calculate densitometry.

Statistical analysis. All experimental images were obtained from at least three rats in each group independently, and representative images were selected and exhibited in this article. The experimental data obtained from at least six rats in each group were analyzed with SPSS software (version 21.0; SPSS, Inc.) and presented as the mean \pm standard deviation (SD). One-way analysis of variance (AVOVA) followed by the Tukey post hoc test was used to analyze the significance of differences between groups. $\mathrm{P}<0.05$ was considered to indicate a statistically significant difference.

\section{Results}

Morphology of the left testes. Representative H\&E staining images are presented in Fig. 2. The seminiferous epithelium morphology of the testes from the experimental varicocele group was disrupted at 8 (Fig. 2C) and 12 weeks (Fig. 2D) compared with that in the sham group at 8 (Fig. 2A) and 12 weeks (Fig. 2B), respectively. Disorganization of germ cells, atrophy of seminiferous tubules, excessive space in the testis mesenchyme, reduced numbers of germ cells and mature sperm, and shedding of immature germ cells (black arrowheads in Fig. 2C and D) in the cavity were observed at different stages in the experimental varicocele group. The percentage of tubules containing deciduous immature germ cells is presented in Fig. 2E. The results demonstrated that the experimental varicocele caused a loss of immature germ cells compared with that in the sham group $(\mathrm{P}<0.05)$. The percentage was higher in the experimental varicocele group at 12 weeks than at 8 weeks $(\mathrm{P}<0.05)$.

The ultrastructure of apical ES in the left testes was observed using transmission electron microscopy (Fig. 3). In the sham group at 8 (Fig. 3A) and 12 weeks (Fig. 3B), the heads of elongating spermatids (black asterisk in Fig. 3A and B) were oriented towards the basement membrane, and the elongating spermatids were tightly attached to Sertoli cells via the apical ES. The apical ES in the normal rat testis (Fig. 3A and B) was characterized by a testis-specific structure, which was only observed in Sertoli cells. The actin filament bundles (black arrowhead) were sandwiched between the endoplasmic reticulum (ER) and the Sertoli cell membrane. In contrast, in rat testes in the experimental varicocele groups at 8 (Fig. 3C) and 12 weeks (Fig. 3D) following surgery, the structure of the apical ES was disrupted, and the elongating spermatids showed loss of orientation toward the basement membrane (black asterisk in Fig. 3C and D). Furthermore, the actin filament bundles (black arrowhead in Fig. 3C and D) and ER, important component structures of the apical ES, were disorganized or even absent from their proper position.

Dysregulation of cell cycle in left testicular cells from experimental varicocele rats. As presented in Fig. 4E, the experimental varicocele resulted in deregulation of the cell cycle in the left testes. The percentages of cells in the $\mathrm{G}_{1}$ and $\mathrm{G}_{2} / \mathrm{M}$ phases in the experimental varicocele group (Fig. 4C and D) were lower than those in the sham group (Fig. 4A and $\mathrm{B} ; \mathrm{P}<0.05$ ) and the percentage of cells in the $\mathrm{S}$ phase in the experimental varicocele group was higher than that in the sham group $(\mathrm{P}<0.05)$.

Expression of AJ component proteins in the left testes of rats. The expression levels of AJ structural proteins, i.e., $\mathrm{N}$-cadherin, E-cadherin, $\alpha$-catenin, $\beta$-catenin and $\gamma$-catenin, were analyzed using western blotting (Fig. 5A). The results (Fig. 5B) indicated that the varicocele resulted in a decrease in the expression of AJ structural proteins $(\mathrm{P}<0.05)$.

Changes in FAK phosphorylation and ERK signal activation. The levels of FAK, p-FAK-Tyr ${ }^{397}$, Src, p-Src-Tyr ${ }^{416}$, ERK1/2 and p-ERK1/2 were analyzed using western blotting (Fig. 6A and B). The experimental varicocele increased the levels of p-FAK-Tyr ${ }^{397}$, p-Src-Tyr ${ }^{416}$ and p-ERK1 $(\mathrm{P}<0.05)$.

\section{Discussion}

During spermatogenesis, germ cells differentiate from the spermatogonium to mature spermatids and must undergo a series of complicated biological processes (31). The AJs between Sertoli cells and germ cells function as anchors and supply nutrients, and also provide an important platform for signal transduction involved in the cell cycle, seminiferous epithelium cycle, cell differentiation and cell communication (6). Notably, 

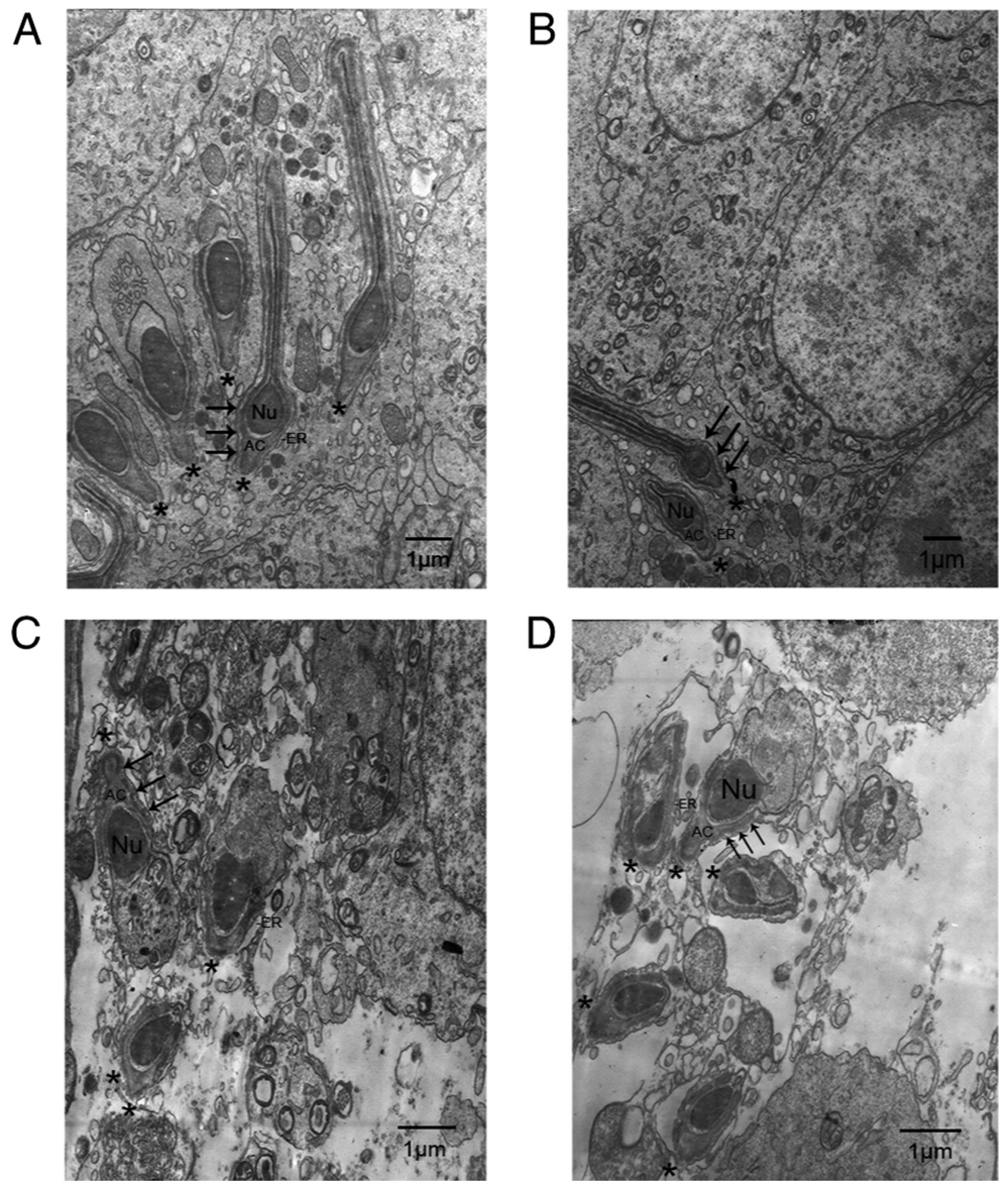

Figure 3. Ultrastructure of apical ES in the left testes. Sham group for the (A) 8 and (B) 12 weeks, and the experimental varicocele group for the (C) 8 and (D) 12 weeks. The normal apical ES is shown in A and B on the sides of Sertoli cells, as typified by orderly actin filament bundles (black arrowhead) sandwiched between the ER and Sertoli cell membrane. The heads of elongating spermatids in the sham group (black asterisks in A and B) maintained their orientations and pointed towards the basement membrane of seminiferous tubules. The structure of the apical ES in C and D was damaged, as shown by the misorientation of elongating spermatid heads (black asterisk) and the disorganization and loss of actin filament bundles (black arrowhead) and the ER. These results are representative of three rats from each group. ES, ectoplasmic specialization; ER, endoplasmic reticulum; Nu, nucleus; AC, acrosome.

the germ cells must move through the TJ to enter into the adluminal compartment from the basal compartment and finally undergo spermiation (6). Therefore, the AJs existing between Sertoli cells and germ cells are not stable and undergo extensive and consistent assembling and disassembling, leading to an increased sensitivity to microenvironmental change, external stimuli and toxicants (6). The varicocele is a common disease of the male reproductive system and the main cause of male infertility; however, the specific underlying mechanisms have not yet been identified (32). The present study revealed that varicocele caused loss of premature germ cells from the seminiferous epithelium, damage to the AJ ultrastructure, disorientation of spermatid heads, dysregulation of the cell cycle and downregulation of AJ structural proteins (N-cadherin, E-cadherin, $\alpha$-catenin, $\beta$-catenin and $\gamma$-catenin). Taken together, these results supported the disruption of AJs directly or indirectly in the left testes after induction of experimental varicocele. These data provide insights into the pathophysiology of varicocele and will be useful for the development of new treatments and diagnostic techniques for the disease.

Activation of the MAPK and PI3K/AKT signaling pathways is involved in the disruption of AJs and TJs, likely by perturbing the bundling of actin at AJ or TJ sites. This mechanism has been extensively studied with regard to reproductive toxicology induced by various environmental toxicants (e.g., cadmium and bisphenol A) and the effect of cytokines [e.g., transforming growth factor (TGF)- $\beta 3$ and tumor necrosis factor (TNF)- $\alpha$ ] on spermatogenesis in vivo and in vitro. The administration of specific inhibitors targeting the MAPK and PI3K/AKT signaling pathways could delay or block toxicant-induced BTB disruption and immature germ cell loss from the epithelium (13,14,33-38). Over the last decade, studies have shown that the ERK signaling pathway plays a crucial role in actin polymerization and cell adhesion (39-41). Furthermore, ERK regulates the phosphorylation of myosin light-chain kinase and directly affects its biological activity (42). Therefore, the dynamics 

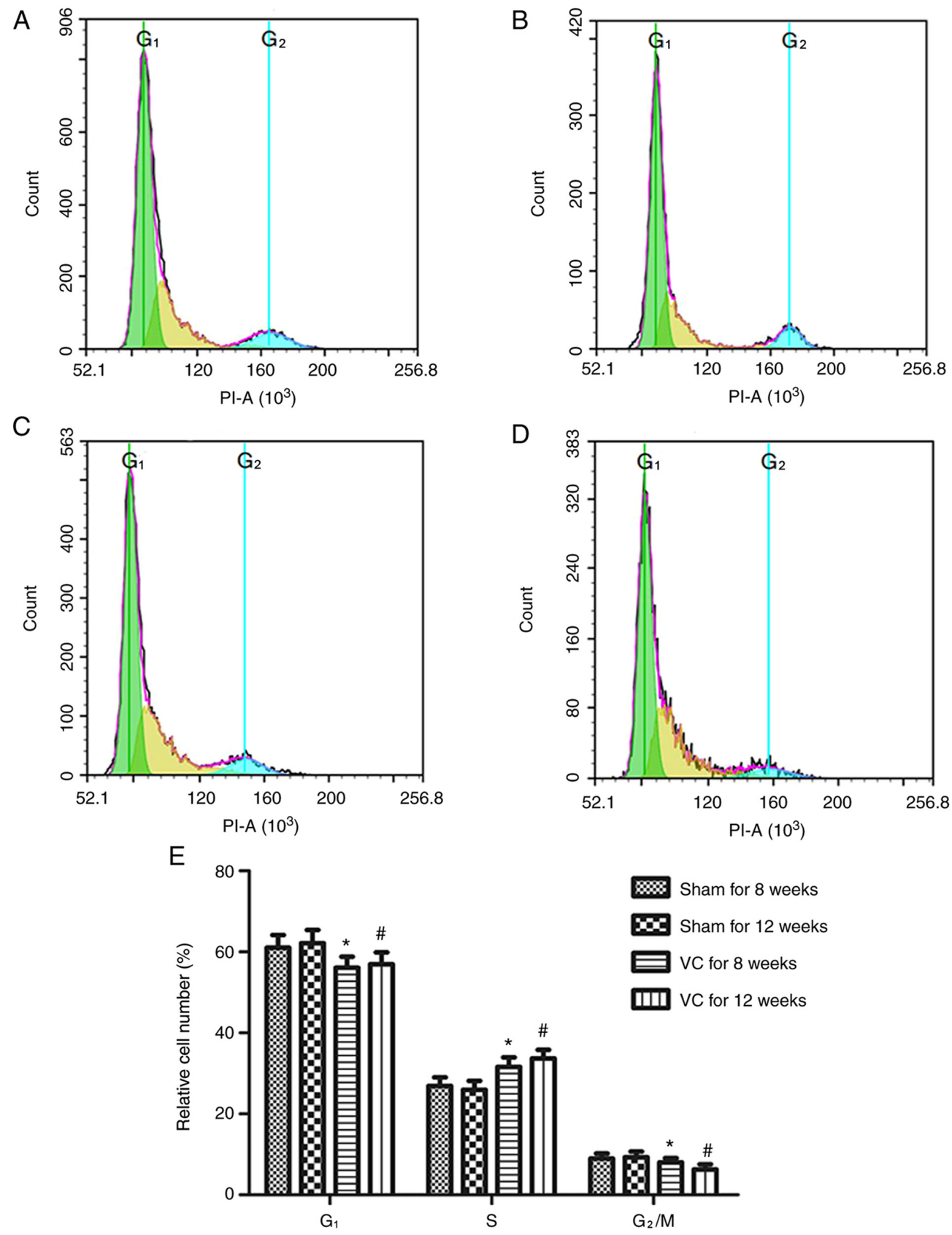

Figure 4. Dysregulation of the cell cycle in left testicular cells. Sham group for the (A) 8 and (B) 12 weeks, and the experimental varicocele group for the (C) 8 and (D) 12 weeks. (E) Percentages of cells in $G_{1}, S$ and $G_{2} / M$ phases in the different groups. The data are presented as the mean $\pm S D(n=6)$. "P<0.05 vs. sham group for 8 weeks; ${ }^{\#} \mathrm{P}<0.05$ vs. sham group for 12 weeks. VC, varicocele.

of actin-based AJs are closely associated with the activation of ERK. The results of the present study demonstrated that varicocele increased the phosphorylation of ERK1, indicating that the ERK signaling pathway was activated in the left testes of rats in the experimental varicocele model; this may be associated with the disruption of AJs, the loss of immature germ cells and disorders of the cell cycle. Furthermore, experimental varicocele increased the levels of $\mathrm{p}-\mathrm{FAK}-\mathrm{Tyr}^{397}$ in the left testes of rats. This is the only phosphorylated form of FAK and provides a combined site for binding of various signaling molecules, such as the SH2 domains of Src (20) and PI3K (21), which are involved in integrin-initiated signaling pathways at the plasma membrane. Varicocele also increased levels of $\mathrm{p}$-Src-Tyr ${ }^{416}$, which is the active form of
Src and the most important signaling molecule downstream of p-FAK-Tyr ${ }^{397}$, in the left testes of experimental rats.

Due to the importance of FAK in the regulation of AJ dynamics and its various biological activities in other epithelial tissues or cell migration, the results of the present study suggested that activation of the ERK signaling pathway may be stimulated, at least in part, by increased phosphorylation of FAK at $\mathrm{Tyr}^{397}$ and through three putative pathways involving integrins. Specifically, following autophosphorylation of FAK-Tyr ${ }^{397}$ at apical ES sites, p-FAK-Tyr ${ }^{397}$ recruits Src (20) to form the activated FAK-Src complex, and then recruits and activates the p130Cas (Crk-associated substrate)/Crk complex. The latter utilizes Ras to activate the Raf-1/MEK/ERK signaling pathway (43). Alternatively, the activated FAK/Src complex 


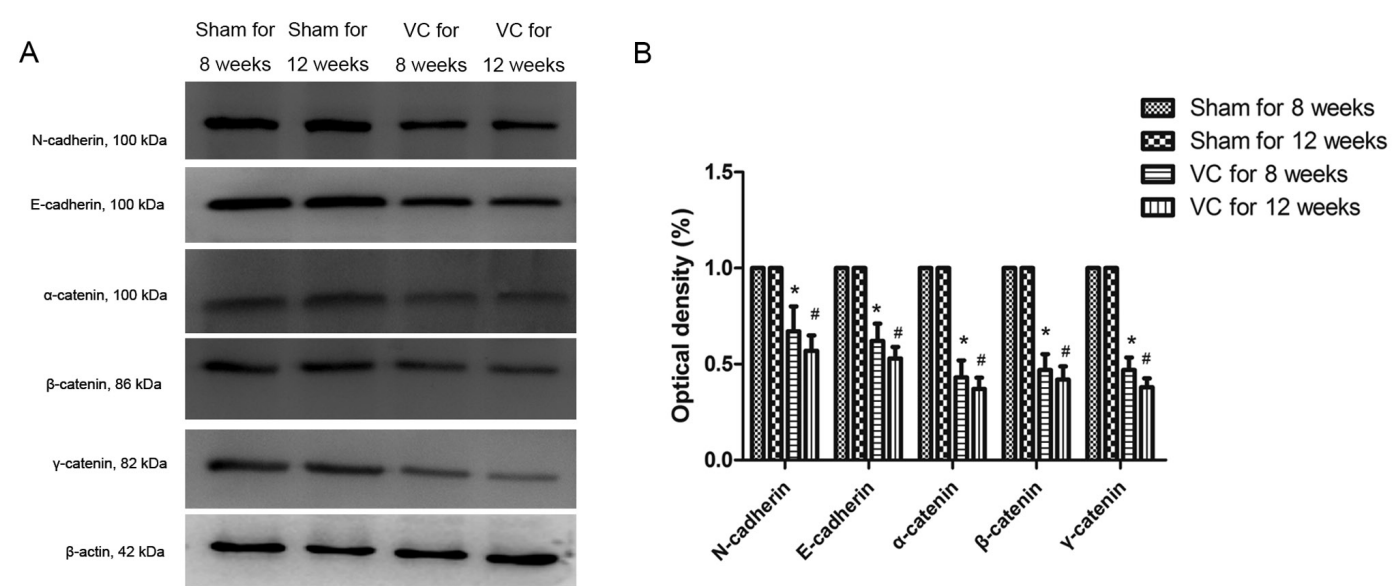

Figure 5. Expression of AJ structural proteins. (A) Immunoblot analysis of AJ structural proteins. (B) Summary of immunoblotting results. Western blot analysis was used to assess the expression of AJ structural proteins ( $\mathrm{N}$-cadherin, E-cadherin, $\alpha$-catenin, $\beta$-catenin and $\gamma$-catenin) in the different groups. The data are presented as the mean $\pm \mathrm{SD}(\mathrm{n}=3) .{ }^{*} \mathrm{P}<0.05$ vs. sham group for 8 weeks; ${ }^{*} \mathrm{P}<0.05$ vs. sham group for 12 weeks. AJ, anchoring junction; VC, varicocele.

A

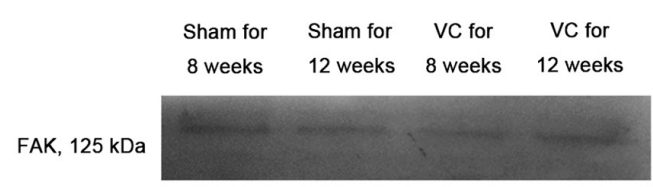

p-FAK-Tyr ${ }^{397}, 125 \mathrm{kDa}$

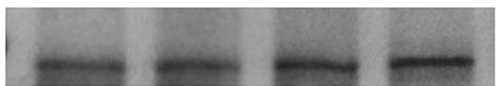

$\mathrm{Src}, 60 \mathrm{kDa}$

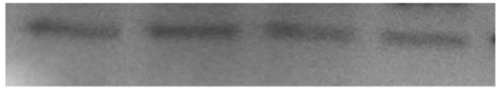

p-Src-Tyr ${ }^{416}, 60 \mathrm{kDa}$

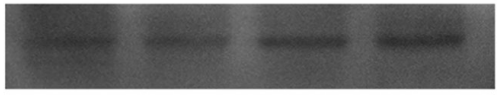

ERK1, $44 \mathrm{kDa}$ ERK2, $42 \mathrm{kDa}$

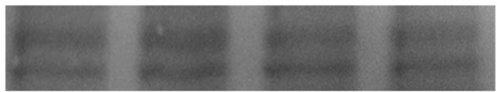

p-ERK1, $44 \mathrm{kDa}$ p-ERK2, $42 \mathrm{kDa}$

$\beta$-actin, $42 \mathrm{kDa}$


B
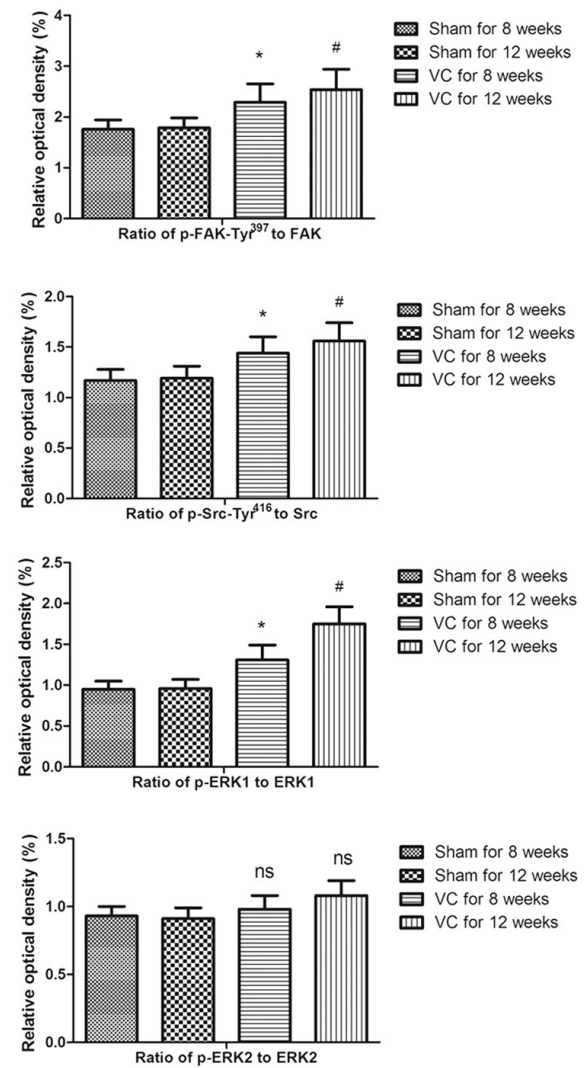

Figure 6. Expression of AJ-associated signaling molecules. (A) Immunoblot analysis of AJ-associated signaling molecules. (B) Summary of immunoblotting results. The levels of FAK, p-FAK-Tyr ${ }^{397}$, Src, p-Src-Tyr ${ }^{416}$, ERK1/2 and p-ERK1/2 were investigated using western blotting. The data are presented as the mean $\pm \mathrm{SD}(\mathrm{n}=3)$. ${ }^{*} \mathrm{P}<0.05$ vs. the sham group for 8 weeks; ${ }^{*} \mathrm{P}<0.05$ vs. the sham group for 12 weeks; ns, not significant; AJ, anchoring junction; $\mathrm{p}$, phosphorylated; $\mathrm{VC}$, varicocele; FAK, focal adhesion kinase; ERK, extracellular signal-regulated protein kinase.

phosphorylates FAK at $\mathrm{Tyr}^{925}$, and p-FAK-Tyr ${ }^{925}$ then recruits Grb2,partly contributing to the activation of the Raf-1/MEK/ERK signaling pathway (39). Finally, p-FAK-Tyr ${ }^{397}$ at the apical ES sequentially interacts with PI3K p $85 \alpha$ and PI3K p110; the complex then recruits and activates Akt, resulting in the activation of the PI3K/Akt signaling cascade (44). In addition, Akt can phosphorylate p21-activated kinase (PAK). Activation of PAK results in the phosphorylation of Raf- 1 and activation of the Raf-1/MEK/ERK signaling pathway (45). In addition, our previous study showed that experimental varicocele increased TGF- $\beta 3$ and TNF- $\alpha$ levels in the left testes of rats, which may also contribute to the activation of the MAPK signaling pathway (46).

FAK is a non-receptor protein tyrosine kinase that is highly expressed at the apical ES and BTB in the testes and is an important regulator of TJ and AJ dynamics (7). Distribution of different forms of phosphorylated FAK (e.g., p-FAK-Tyr ${ }^{397}$ and $\mathrm{p}-\mathrm{FAK}-\mathrm{Tyr}^{407}$ ) is spatial and temporal at particular sites in 
specific stages of the epithelium cycle, suggesting an intimate connection with cell junction dynamics. Notably, p-FAK-Tyr ${ }^{397}$ is the only autophosphorylated form of FAK. In a number of other epithelial tissues, FAK is the main component of focal adhesion complexes, which act as anchoring devices between the cells and matrix. After being activated by integrin or growth factor receptors, $\mathrm{Tyr}^{397}$ is autophosphorylated and mediates signal transduction to the actin-based cytoskeleton or activates signaling pathways by interacting with downstream signaling molecules, such as related kinases, adaptor proteins, guanine nucleotide exchanging factors and small GTPases (e.g., Ras, Rac and Rho) (47). In the present study, increased phosphorylation of FAK at $\mathrm{Tyr}^{397}$ in the testes of rats with experimental varicocele may have disrupted AJs through mechanisms other than mediating the activation of ERK1 signaling, as aforementioned. For example, FAK regulates the polymerization of actin mediated by Arp 2/3, which is realized by preventing the nuclear translocation of Arp 2/3 activator [namely, the neuronal Wiskott-Aldrich syndrome protein (N-WASP)] by increasing FAK-mediated phosphorylation and activating Arp 2/3 through direct interaction between the FAK 4.1/ezrin/radixin/moesin domain and Arp 3 (19). In addition, the direct interaction between FAK and Arp3 is negatively regulated by p-FAK-Tyr ${ }^{397}$ (19), leading to disorganization of actin polymerization, followed by disruption of AJs and TJs (48-50). In contrast, the apical ES and BTB may work as a functional unit, namely, the apical ES-BTB functional axis, due to the opening of TJs, which permits preleptotene and leptotene spermatocytes to cross the BTB, and the degeneration of apical ES during spermiation both occur during stage VIII of the seminiferous epithelium cycle (51). In addition, the association between apical ES and BTB may occur as a result of the recycling of p-FAK-Tyr ${ }^{397}$. After the release of mature spermatids at stage VIII, p-FAK-Tyr ${ }^{397}$ at the apical ES is transferred to the cytoplasm, and high levels of p-FAK-Tyr ${ }^{397}$ in the cytoplasm may induce the opening of the BTB (19). In the present study, increased levels of $\mathrm{p}-\mathrm{FAK}-\mathrm{Tyr}^{397}$ in the left testes of rats with experimental varicocele may have altered the balance between the apical ES-BTB functional axis, resulting in disruption of the AJ.

Regulation of cell junction (e.g., TJ, apical ES and basal ES) dynamics in the testis is a complicated biological process that involves the transduction of multiple signaling molecules and is not yet fully understood. Disruption of AJs in the left testes of rats with experimental varicocele may be the primary or secondary damage induced by varicocele. Further studies are required in order to assess the other inducers and elucidate the specific mechanisms. In addition, the exact mechanisms inducing FAK-Tyr ${ }^{397}$ phosphorylation, ERK activation and AJ structural protein downregulation are unclear. Nevertheless, the loss of premature germ cells and enhancement of p-FAK-Tyr ${ }^{397}$ levels in the model used in the present study suggested that protein kinase inhibitors of FAK-Tyr ${ }^{397}$ or inhibitors of the ERK signaling pathway could be used in the treatment of varicocele to prevent the loss of premature spermatids and improve fertility. Furthermore, the results of the present study also implied that FAK-Tyr ${ }^{397}$ phosphorylation could be used to prevent the formation of mature spermatids for male contraception.

In summary, varicocele resulted in disruption of the structure and function of AJs in the left testes of rats. To a certain extent, the increased $\mathrm{p}-\mathrm{FAK}-\mathrm{Tyr}^{397}$ levels may have contributed to AJ damage, potentially by activating the ERK1 signaling pathway, disturbing the actin-based filament network and disrupting the balance of the apical ES-BTB functional axis, which is crucial for understanding the pathological mechanisms of varicocele that contribute to male infertility and for identifying new therapeutic targets for varicocele. Nevertheless, due to the sophisticated structure and function of AJ, the complicated pathological mechanisms of varicocele, as well as the limitation that all the results were gained from the in vivo study, the upregulated p-FAK-Tyr ${ }^{397}$ levels and the activation of ERK1 signaling pathway were perhaps not directly and necessarily associated with the disruption of AJs in experimental rat testes, and at least were not the only inducements. Future studies will be designed and performed that include both in vitro and in vivo experiments in order to verify the exact mechanism and investigate these hypotheses. For example, utilizing the Sertoli cells and germ cells co-culture system, then increasing or decreasing FAK phosphorylation at $\mathrm{Tyr}^{397}$, or activating or inhibiting the ERK1 signaling pathway to evaluate the structure and function of AJs.

\section{Acknowledgements}

Not applicable.

\section{Funding}

The present study was funded by the Training Programme Foundation for the Talents of Health and Family Planning Commission in Fujian Province (grant no. 2018-1-74), the Start-up Foundation of Fujian Medical University (grant no. 2017XQ1005), the Research Foundation for High-level Talents of Fujian Medical University (grant no. XRCZX2017033), the Natural Science Foundation of Fujian Province (grant no. 2017J01819), and the Co-construction Science Foundation of National Health and Family Planning Commission-Joint Program for Tackling Key Problems of Health and Education in Fujian Province (grant no. WKJ2016-2-35).

\section{Availability of data and materials}

The datasets used and/or analyzed during the current study are available from the corresponding authors on reasonable request.

\section{Authors' contributions}

LZ performed the experiments, conducted the statistical analysis and drafted the manuscript. WW conceived the study, performed the experiments and helped draft the manuscript. $\mathrm{XZ}$ participated in the design and coordination of the study and helped draft the manuscript. LZ and WW confirm the authenticity of all the raw data. All authors have read and approved the final manuscript.

\section{Ethics approval and consent to participate}

The experimental procedures were performed in accordance with the Guidelines for the Care and Use of Laboratory 
Animals approved by Fujian Medical University (Animal Approval Committee no. \#SYXK-2012-0001).

\section{Patient consent for publication}

Not applicable.

\section{Competing interests}

The authors declare that they have no competing interests.

\section{References}

1. Russell L: Observations on rat Sertoli ectoplasmic ('junctional') specializations in their association with germ cells of the rat testis. Tissue Cell 9: 475-498, 1977.

2. Siu MK and Cheng CY: Dynamic cross-talk between cells and the extracellular matrix in the testis. BioEssays 26: 978-992, 2004.

3. Vogl AW, Pfeiffer DC, Mulholland D, Kimel G and Guttman J: Unique and multifunctional adhesion junctions in the testis: Ectoplasmic specializations. Arch Histol Cytol 63: 1-15, 2000.

4. Wong EW, Mruk DD and Cheng CY: Biology and regulation of ectoplasmic specialization, an atypical adherens junction type, in the testis. Biochim Biophys Acta 1778: 692-708, 2008.

5. Siu MK, Wong CH, Xia W, Mruk DD, Lee WM and Cheng CY: The $\beta 1$-integrin-p-FAK-p130Cas-DOCK180-RhoA-vinculin is a novel regulatory protein complex at the apical ectoplasmic specialization in adult rat testes. Spermatogenesis 1: 73-86, 2011

6. Cheng CY and Mruk DD: Cell junction dynamics in the testis: Sertoli-germ cell interactions and male contraceptive development. Physiol Rev 82: 825-874, 2002.

7. Siu ER, Wong EW, Mruk DD, Porto CS and Cheng CY: Focal adhesion kinase is a blood-testis barrier regulator. Proc Natl Acad Sci USA 106: 9298-9303, 2009

8. Gungor-Ordueri NE, Mruk DD, Wan HT, Wong EW, Celik-Ozenci C, Lie PP and Cheng CY: New insights into FAK function and regulation during spermatogenesis. Histol Histopathol 29: 977-989, 2014.

9. Daniel JM and Reynolds AB: Tyrosine phosphorylation and cadherin/catenin function. BioEssays 19: 883-891, 1997.

10. Wine RN and Chapin RE: Adhesion and signaling proteins spatiotemporally associated with spermiation in the rat. J Androl 20: 198-213, 1999.

11. Fashena SJ and Thomas SM: Signalling by adhesion receptors. Nat Cell Biol 2: E225-E229, 2000.

12. Hamaguchi M, Matsuyoshi N, Ohnishi Y, Gotoh B, Takeichi M and Nagai Y: p60v-src causes tyrosine phosphorylation and inactivation of the N-cadherin-catenin cell adhesion system. EMBO J 12: 307-314, 1993

13. Cheng CY, Wong EW, Lie PP, Li MW, Su L, Siu ER, Yan HH, Mannu J, Mathur PP, Bonanomi M, et al: Environmental toxicants and male reproductive function. Spermatogenesis 1: 2-13, 2011.

14. Siu MK, Wong CH, Lee WM and Cheng CY: Sertoli-germ cell anchoring junction dynamics in the testis are regulated by an interplay of lipid and protein kinases. J Biol Chem 280 : 25029-25047, 2005

15. Siu ER, Wong EW, Mruk DD, Sze KL, Porto CS and Cheng CY: An occludin-focal adhesion kinase protein complex at the blood-testis barrier: A study using the cadmium model. Endocrinology 150: 3336-3344, 2009.

16. Quadri SK: Cross talk between focal adhesion kinase and cadherins: Role in regulating endothelial barrier function. Microvasc Res 83: 3-11, 2012.

17. Su L, Mruk DD, Lui WY, Lee WM and Cheng CY: P-glycoprotein regulates blood-testis barrier dynamics via its effects on the occludin/zonula occludens 1 (ZO-1) protein complex mediated by focal adhesion kinase (FAK). Proc Natl Acad Sci USA 108: 19623-19628, 2011.

18. Wan HT, Mruk DD, Wong CK and Cheng CY: Perfluorooctanesulfonate (PFOS) perturbs male rat Sertoli cell blood-testis barrier function by affecting F-actin organization via p-FAK-Tyr(407): An in vitro study. Endocrinology 155: 249-262, 2014
19. Lie PP, Mruk DD, Mok KW, Su L, Lee WM and Cheng CY: Focal adhesion kinase-Tyr ${ }^{407}$ and $-\mathrm{Tyr}^{397}$ exhibit antagonistic effects on blood-testis barrier dynamics in the rat. Proc Natl Acad Sci USA 109: 12562-12567, 2012.

20. Eide BL, Turck CW and Escobedo JA: Identification of Tyr-397 as the primary site of tyrosine phosphorylation and pp60sre association in the focal adhesion kinase, pp125FAK. Mol Cell Biol 15: 2819-2827, 1995.

21. Parsons JT: Focal adhesion kinase: The first ten years. J Cell Sci 116: 1409-1416, 2003.

22. Fretz PC and Sandlow JI: Varicocele: Current concepts in pathophysiology, diagnosis, and treatment. Urol Clin North Am 29: 921-937, 2002.

23. Bach PV, Najari BB and Goldstein M: Varicocele - a case for early intervention. F1000 Res 5: 5, 2016.

24. Luo DY, Yang G, Liu JJ, Yang YR and Dong Q: Effects of varicocele on testosterone, apoptosis and expression of StAR mRNA in rat Leydig cells. Asian J Androl 13: 287-291, 2011.

25. Chiba K, Ramasamy R, Lamb DJ and Lipshultz LI: The varicocele: Diagnostic dilemmas, therapeutic challenges and future perspectives. Asian J Androl 18: 276-281, 2016.

26. Saypol DC, Howards SS, Turner TT and Miller ED Jr: Influence of surgically induced varicocele on testicular blood flow, temperature, and histology in adult rats and dogs. J Clin Invest 68: 39-45, 1981

27. Pastuszak AW and Wang R: Varicocele and testicular function. Asian J Androl 17: 659-667, 2015.

28. Ozbek E, Yurekli M, Soylu A, Davarci M and Balbay MD: The role of adrenomedullin in varicocele and impotence. BJU Int 86: 694-698, 2000.

29. Vyas A, Ram H, Purohit A and Jatwa R: Adverse effects of subchronic dose of aspirin on reproductive profile of male rats. J Pharm (Cairo) 2016: 6585430, 2016.

30. Rotgers E, Cisneros-Montalvo S, Jahnukainen K, Sandholm J, Toppari J and Nurmio M: A detailed protocol for a rapid analysis of testicular cell populations using flow cytometry. Andrology 3 : 947-955, 2015.

31. Neto FT, Bach PV, Najari BB, Li PS and Goldstein M: Spermatogenesis in humans and its affecting factors. Semin Cell Dev Biol 59: 10-26, 2016.

32. Khera M and Lipshultz LI: Evolving approach to the varicocele. Urol Clin North Am 35: 183-189, 2008.

33. Lui WY, Wong CH, Mruk DD and Cheng CY: TGF-beta3 regulates the blood-testis barrier dynamics via the p38 mitogen activated protein (MAP) kinase pathway: An in vivo study. Endocrinology 144: 1139-1142, 2003.

34. Li MW, Xia W, Mruk DD, Wang CQ, Yan HH, Siu MK, Lui WY, Lee WM and Cheng CY: Tumor necrosis factor \{alpha\} reversibly disrupts the blood-testis barrier and impairs Sertoli-germ cell adhesion in the seminiferous epithelium of adult rat testes. J Endocrinol 190: 313-329, 2006.

35. Li MW, Mruk DD, Lee WM and Cheng CY: Disruption of the blood-testis barrier integrity by bisphenol $\mathrm{A}$ in vitro: Is this a suitable model for studying blood-testis barrier dynamics? Int J Biochem Cell Biol 41: 2302-2314, 2009.

36. Thuillier R, Manku G, Wang Y and Culty M: Changes in MAPK pathway in neonatal and adult testis following fetal estrogen exposure and effects on rat testicular cells. Microsc Res Tech 72: 773-786, 2009.

37. Wong CH, Mruk DD, Siu MK and Cheng CY: Blood-testis barrier dynamics are regulated by \{alpha\}2-macroglobulin via the c-Jun N-terminal protein kinase pathway. Endocrinology 146: 1893-1908, 2005.

38. Wong CH, Mruk DD, Lui WY and Cheng CY: Regulation of blood-testis barrier dynamics: An in vivo study. J Cell Sci 117: 783-798, 2004

39. Hirata H, Gupta M, Vedula SR, Lim CT, Ladoux B and Sokabe M: Quantifying tensile force and ERK phosphorylation on actin stress fibers. Methods Mol Biol 1487: 223-234, 2017.

40. Mendoza MC, Vilela M, Juarez JE, Blenis J and Danuser G: ERK reinforces actin polymerization to power persistent edge protrusion during motility. Sci Signal 8: ra47, 2015.

41. Logue JS, Cartagena-Rivera AX, Baird MA, Davidson MW, Chadwick RS and Waterman CM: Erk regulation of actin capping and bundling by Eps8 promotes cortex tension and leader bleb-based migration. eLife 4: e08314, 2015.

42. Klemke RL, Cai S, Giannini AL, Gallagher PJ, de Lanerolle P and Cheresh DA: Regulation of cell motility by mitogen-activated protein kinase. J Cell Biol 137: 481-492, 1997.

43. Cary LA and Guan JL: Focal adhesion kinase in integrin mediated signaling. Frontiers in bioscience. Front Biosci 4: D102-D113, 1999. 
44. Foster FM, Traer CJ, Abraham SM and Fry MJ: The phosphoinositide (PI) 3-kinase family. J Cell Sci 116: 3037-3040, 2003.

45. Kumar R and Vadlamudi RK: Emerging functions of p21-activated kinases in human cancer cells. J Cell Physiol 193: 133-144, 2002.

46. Tomar A and Schlaepfer DD: Focal adhesion kinase: Switching between GAPs and GEFs in the regulation of cell motility. Curr Opin Cell Biol 21: 676-683, 2009.

47. Schaller MD: Cellular functions of FAK kinases: Insight into molecular mechanisms and novel functions. J Cell Sci 123 1007-1013, 2010

48. Goley ED and Welch MD: The ARP2/3 complex: An actin nucleator comes of age. Nat Rev Mol Cell Biol 7: 713-726, 2006.
49. Wu X, Suetsugu S, Cooper LA, Takenawa T and Guan JL: Focal adhesion kinase regulation of N-WASP subcellular localization and function. J Biol Chem 279: 9565-9576, 2004.

50. Serrels B, Serrels A, Brunton VG, Holt M, McLean GW, Gray CH, Jones GE and Frame MC: Focal adhesion kinase controls actin assembly via a FERM-mediated interaction with the Arp2/3 complex. Nat Cell Biol 9: 1046-1056, 2007.

51. Yan HH, Mruk DD, Wong EW, Lee WM and Cheng CY: An autocrine axis in the testis that coordinates spermiation and blood-testis barrier restructuring during spermatogenesis. Proc Natl Acad Sci USA 105: 8950-8955, 2008.

(i) (9) This work is licensed under a Creative Commons EY No No Attribution-NonCommercial-NoDerivatives 4.0 International (CC BY-NC-ND 4.0) License. 